\title{
A Two-component Regulatory System Involved in Clavulanic Acid Production
}

\author{
Hum Nath Jnawali, Tae-Jin Oh, Kwangkyoung Liou, Byoung Chul Park, \\ Jae Kyung Sohng
}

Received: July 10, 2008 / Accepted: October 21, 2008

(C) Japan Antibiotics Research Association

\begin{abstract}
A pair of genes encoding the bacterial twocomponent regulatory system, $\operatorname{orf} 22$ and $\operatorname{orf} 23$, was found next to the clavulanic acid gene cluster of Streptomyces clavuligerus NRRL3585. Orf23 was deleted for the construction of $S$. clavuligerus/Dorf23. Although growth and morphological analyses showed no differences between S. clavuligerus/Dorf23 and wild-type, the production

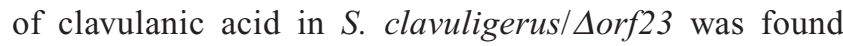
to be decreased. In addition, the co-overexpression of

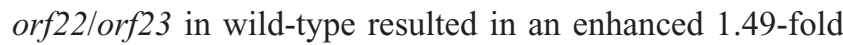
production of clavulanic acid, and the complementation of orf22/orf23 in S. clavuligerus/Dorf23 restored clavulanic acid production about $80 \%$ as normal levels. These results demonstrate that the orf $22 /$ orf 23 two-component regulatory system participates as a positive regulator of the biosynthesis of clavulanic acid and increased levels of $\operatorname{orf} 22 / \operatorname{orf} 23$ can contribute to enhanced production of clavulanic acid in $S$. clavuligerus.
\end{abstract}

Keywords clavulanic acid, histidine kinase, $\beta$-lactam antibiotics, response regulator, Streptomyces clavuligerus, two-component regulatory system

\section{Introduction}

Bacteria sense and respond to a wide variety of signals through a complex network of signaling systems, many of which are two-component phosphotransfer pathways. Twocomponent systems (TCS) are a family of proteins that mediate the adaptation to changing environments by modifying the phosphorylated state of a pair of proteins: a sensor kinase and a response regulator (RR). These systems have been shown to be involved in a variety of bacterial cellular responses, such as chemotaxis, sporulation, photosynthesis, osmoregulation, antibiotic production, and pathogenicity $[1 \sim 3]$. Sensors are usually integral membrane proteins that respond to particular chemical and/or physical cues by modifying the phosphorylated state of their cognate RR. Sensors become phosphorylated at a conserved histidine residue, and this phosphate group is then transferred to a conserved aspartate residue in the regulator. The sensor kinase and RR pairs that mediate these signaling processes are referred to as two-component signal transduction systems [4]. The TCS normally organize as pairs on the genome with the RR and sensor kinase (histidine kinase, HK) following to each other in the transcription direction. TCS-RR, which respond to changes in environmental factors, also influence antibiotic production [5].

Streptomyces clavuligerus NRRL3585 is a Grampositive filamentous bacterium that is well known for its ability to produce an array of $\beta$-lactam compounds, including cephamycin $\mathrm{C}$, clavulanic acid (CA) and other
J. K. Sohng (Corresponding author), H. N. Jnawali, T.-J. Oh, K. Liou: Institute of Biomolecule Reconstruction (iBR), Department of Pharmaceutical Engineering, SunMoon University, \#100, Asansi, Chungnam 336-708, Korea,

E-mail: sohng@sunmoon.ac.kr
B. C. Park: Korea Research Institute of Bioscience and Biotechnology, Daejeon, Korea 


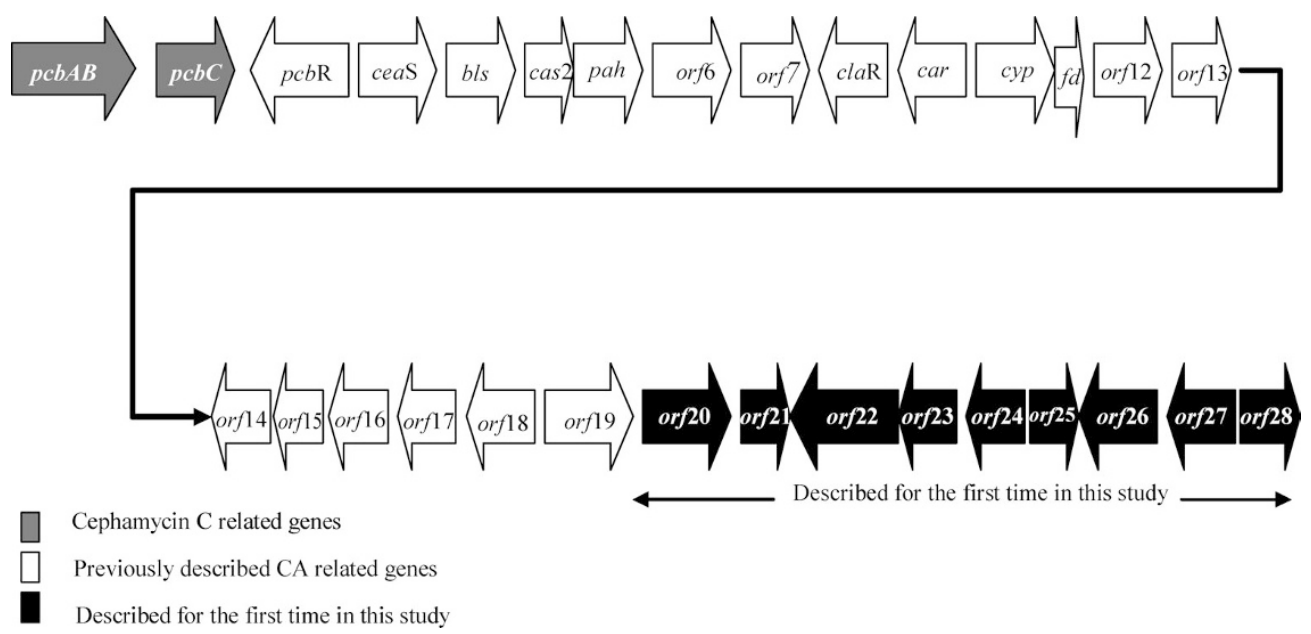

Fig. 1 CA gene cluster with newly described downstream genes.

structurally related clavams. CA has poor antibacterial activity, but it binds irreversibly to the serine hydroxyl group at the active center of $\beta$-lactamase, producing a stable acylated intermediate and resulting in the inactivation of the enzyme [6]. The $c c a R$, which controls the production of both cephamycin $\mathrm{C}$ and $\mathrm{CA}$, has been found in the cephamycin $\mathrm{C}$ gene cluster [7]. The CA gene cluster contains claR, which encodes a LysR-type regulatory protein that controls $\mathrm{CA}$ production $[8,9]$. Amplification of the claR on a multicopy plasmid resulted in a 3-fold increase in CA production, whereas cephamycin production was significantly reduced [8]. Recently it was reported that Pah2 catalyzes the reaction from guanidinoproclavaminate to proclavaminic acid, where the guanidine group from the arginine-derived end of the molecule is removed, and also a single additional copy of pah2 introduced into chromosome via an integrative plasmid showed enhanced production of CA [10].

In this study, the effects of the TCS genes orf 22 and orf23, which are downstream of the CA gene clusters from S. clavuligerus NRRL3585, were examined by disrupting orf23 (Fig. 1). We also examined the effects of overexpression of $\operatorname{orf} 22$ and $\operatorname{orf} 23$ on the production of CA. For overexpression, we cloned orf 22 and orf 23 genes together with a pIBR25 containing strong promoter, ermE*. Although the functions of the majority of the TCS genes remain unknown, the existence of TCS in S. clavuligerus NRRL3585 implies that it may have important physiological roles. It is first time to identify a complete pair in the downstream region of CA gene cluster. This work demonstrates that the orf $22 / \operatorname{orf} 23$ encoding TCS-RR controls CA production.

\section{Materials and Methods}

\section{Bacterial Strains, Culture Conditions and DNA Manipulation}

Escherichia coli were grown in Luria-Bertani (LB) broth and maintained on LB agar medium at $37^{\circ} \mathrm{C}$. DNA manipulation was carried out in $E$. coli XL1-Blue (Stratagene). Plasmids were propagated in E. coli ET12567 to obtain unmethylated DNA for transformation into $S$. clavuligerus NRRL3585. S. clavuligerus was cultured on different media for different purposes. For protoplast transformation, TSB ( $25 \mathrm{ml}, 1.7 \%$ tryptone, $0.3 \%$ soytone, $0.25 \%$ dextrose, $0.5 \% \mathrm{NaCl}$, and $\left.0.25 \% \quad \mathrm{KH}_{2} \mathrm{PO}_{4}\right)$ supplemented with $1.0 \%(\mathrm{w} / \mathrm{v})$ maltose was initially inoculated with seed culture of $S$. clavuligerus at $28^{\circ} \mathrm{C}$ for 24 hours. The growing seed culture was then transferred to R2YE medium (50 ml, 5.0\% sucrose, $0.02 \% \mathrm{~K}_{2} \mathrm{SO}_{4}, 1.0 \%$ $\mathrm{MgCl}_{2}, 1.0 \%$ glucose, $0.5 \%$ yeast extract, and $0.01 \%$ Difco casamino acid) at $250 \mathrm{rpm}$ and $28^{\circ} \mathrm{C}$ for 36 hours. Streptomyces transformants were supplemented with apramycin $(25 \mu \mathrm{g} / \mathrm{ml})$ or neomycin $(20 \mu \mathrm{g} / \mathrm{ml})$. For CA production, media composed of $1.5 \%$ glycerol, $3.0 \%$ tryptic soy broth, $1.0 \%$ peptone and $1.05 \%$ MOPS was cultured at $\mathrm{pH} 6.5$ for up to 120 hours. All bacterial stocks were stored using $30 \%$ glycerol at $-70^{\circ} \mathrm{C}$. The pGEM-TEasy and pGEM-3Zf $(+)$ (Promega) vectors were the routine cloning vectors used for DNA manipulation. pOJ446 was used to construct the genomic library, and pKC1139 [11] was used for the disruption of orf23. The pIBR25 vector [12] was used for the co-overexpression of pICA1 (harboring orf22/orf23) in the wild-type. The plasmids were isolated from $E$. coli using the alkaline lysis 
method and purified using Qiagen ion-exchange columns (Hilden, Germany). Standard methods were used for DNA cloning, plasmid isolation, and restriction enzyme digestion $[11,13]$.

\section{Construction of Cosmid Library and Sequence Analysis} For cosmid library construction, $5.0 \mu \mathrm{g}$ of $S$. clavuligerus chromosomal DNA was partially digested with Sau3AI and ligated into pOJ446 [11], which was pre-treated with HpaI, dephosphorylated, and restricted with BamHI. The ligated mixture was packaged with Gigapack ${ }^{\circledR}$ III Gold packaging extract (Stratagene) and transduced into E. coli XL1-Blue. A pair of primers of cas2 (clavaminate synthase 2), cas2-F (5'-GTG AAG GAT CCA TCG TGT CAT GGC CTC-3') and cas2-R (5'-CCG GGG CCC AGG GCC TCT AGA AGC CG-3') was designed for the polymerase chain reaction (PCR). The amplified cas 2 fragments were used as a probe to screen the cosmid library of $S$. clavuligerus for the isolation of the CA biosynthetic gene cluster. Hybridization was carried out with the probe at $60^{\circ} \mathrm{C}$ for 5 hours in $20 \mathrm{ml}$ of $2 \times \mathrm{SSC}$ [13]. The full sequencing of the cosmid clone and contig assembly was entrusted to Genotech Co. Ltd. (Daejeon, Korea). The sequences were analyzed using FramePlot version 2.3.2 software [14]. DNA and deduced protein sequence homology searches of databases were performed with the BLAST program [15]. Multiple alignments were performed using the ClustalW program [16].

\section{Disruption of orf 23}

The disruption of orf 23 was attempted using a homologous recombination approach with $\mathrm{pKC} 1139$. For the inactivation of orf23 in S. clavuligerus, the upstream fragment, orf23-U (1,500 bp), and the downstream fragment, orf23-D (1,500 bp), were amplified by PCR. The primer pairs were orf23-UF (5'-CCA AGC TTT TGG CCG CCG TTC CCG GC-3'), orf23-UR (5'-CCA TCT AGA CCT GTC TCC TTG TAG GCA CC-3'); and orf23-DF (5' GAC TCT AGA CGG AGG TGG GCC GGG TG-3'), orf23-DR (5'-GCT GAA TTC GAC GGT GCC GTC GTG CGC G-3'). The amplified DNA fragment of orf23-U was digested with HindIII and $X b a \mathrm{I}$ and cloned into the corresponding sites of $\mathrm{pKC} 1139$, resulting in $\mathrm{pUC} 1$. The PCR fragment orf23-D was digested with $X b a \mathrm{I}$ and EcoRI and ligated into the same sites of pUC1 to give pUD. pUD was digested with $X b a \mathrm{I}$ and ligated with the fragment of the neomycin-resistance gene, resulting in $\mathrm{pHN} 2$. In this final construct, pHN2, orf23 was replaced by neo ${ }^{r}$ in-frame. pHN2 was finally transformed into E. coli ET12567 demethylation host and then transformed into wild-type, $S$. clavuligerus NRRL3585, for deletion by a replicative plasmid-mediated homologous recombination. The conditions for protoplast formation, regeneration, and DNA transformation were slightly modified from the methods of Hopwood [11, 17]. After the formation of protoplasts, they were treated with $0.1 \mathrm{mM}$ aurintricarboxylic acid (ATA) (Sigma) for 10 minutes before being mixed with the plasmids. Then, $200 \mu \mathrm{l}$ of $40 \%$ (w/v) polyethylene glycol 1,000 (PEG, Merck-Shuchardt) solution was promptly added followed by a brief period of centrifugation to remove the PEG and resuspension in P-buffer. The transformed protoplasts were then plated on R2YE regeneration plates and incubated at $28^{\circ} \mathrm{C}$. After 24 hours plates were overlaid with a $0.3 \%$ agar solution containing $20 \mu \mathrm{g} / \mathrm{ml}$ of neomycin to get the pHN2 transformants.

\section{Screening for Disruption Mutants S. clavuligerus/ $\Delta o r f 23$ and Southern Blot Hybridization}

After the fifth generation of selection at $37^{\circ} \mathrm{C}, 411$ colonies were picked at random, and each colony was patched out on separate R2YE plates containing neomycin $(20 \mu \mathrm{g} / \mathrm{ml})$ and apramycin $(25 \mu \mathrm{g} / \mathrm{ml})$. Four of them displayed the double-crossover phenotype of neomycin resistance and apramycin sensitivity. The disrupted orf 23 was confirmed by Southern blot hybridization analysis via standard protocols [11]. Hybridization was performed under high stringency conditions $\left(0.5 \times \mathrm{SSC}, 68^{\circ} \mathrm{C}\right)$ as described elsewhere (Roche) by using the probe labeled with Digoxigenin High Prime DNA Labeling and Detection Starter Kit II (Boehringer) and used for Southern blot analysis on Hybond-N nylon membranes (Amersham). A chromogenic method was used for the detection of probes on a blot.

\section{Construction of Recombinant Plasmids and Transformation into Wild-type}

Plasmids pICA1 was constructed according to standard procedures $[12,18]$. The one sets of oligonucleotides used for amplification were orf22/orf23F (5'-CCG GTG TCT $A G A$ AGG AGA CAG GAC A-3'), orf22/orf23R (5'-GGA GAA GCT TGG CGT GAC CGC CTA-3'). The purified PCR products were first inserted into the pGEM-T-Easy vector (Promega) and then orf22/orf23 fragments were inserted into pIBR25 via XbaI/HindIII to yield pICA1. pIBR25 and pICA1 were finally transformed into $E$. coli ET12567 demethylation host and then again transformed into wild-type. It was designated as $S$. clavuligerus/pIBR25 and $S$. clavuligerus/pICA1, respectively. Thus, the pICA1 recombinant plasmid was complemented into the $\Delta$ orf23-

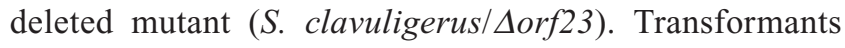
were obtained by $5.0 \mu \mathrm{g} / \mathrm{ml}$ thiostrepton selection. 
Table 1 Deduced functions of the newly described ORFs from downstream CA gene cluster

\begin{tabular}{ccllll}
\hline ORFs & Size (aa) & \multicolumn{1}{c}{ Putative function } & Identity (\%) & Origin & Accession no. \\
\hline orf20 & 393 & Putative cytochrome P450, CypX & 99 & S. clavuligerus & AAR16180 \\
orf21 & 201 & Putative RNA polymerase sigma factor, SigL & 39 & S. coelicolor A3 (2) & NP627178 \\
orf22 & 571 & Putative two-component system histidine kinase & 49 & S. coelicolor A3 (2) & CAC32361 \\
orf23 & 270 & Putative two-component system response regulator & 73 & S. avermitilis & BAC71910 \\
orf24 & 188 & Putative RNA polymerase sigma factor, SigL & 46 & S. coelicolor A3 (2) & NP627178 \\
orf25 & 213 & Hypothetical protein & 26 & S. coelicolor A3 (2) & NP628702 \\
orf26 & 393 & Putative FAD-dependent oxidoreductase & 52 & S. ambofaciens & CAK50855 \\
orf27 & 360 & Putative Ku70/Ku80 protein, Ku1 & 62 & S. avermitilis & BAC70656 \\
orf28 & 181 & Putative DNA ligase, LigD & 68 & S. avermitilis & BAC70657 \\
\hline
\end{tabular}

A.

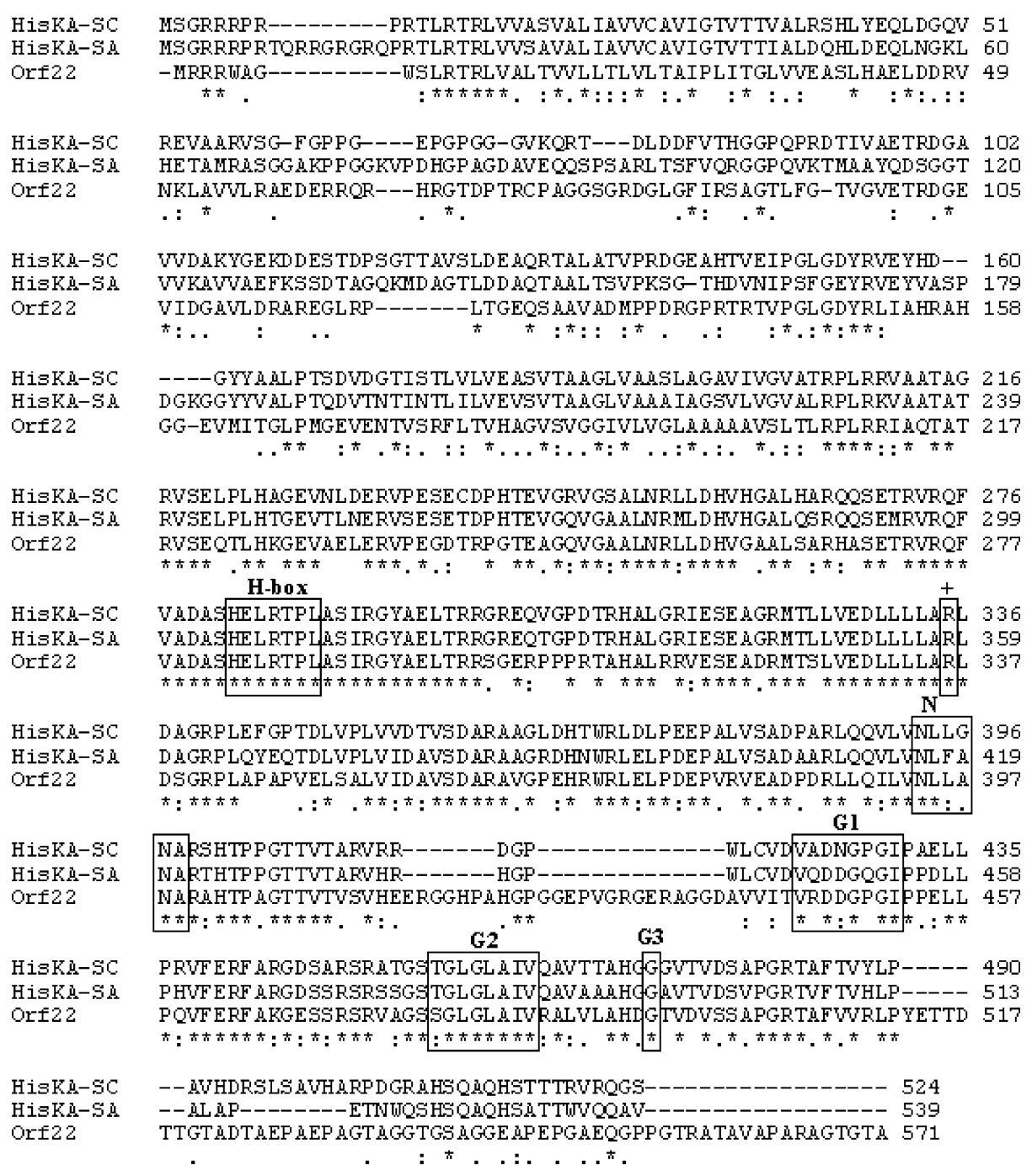

Fig. 2 Amino acid sequence alignment of the TCS-HK (orf22) and TCS-RR (orf23).

A. The deduced amino acid sequences of the $S$. clavuligerus Orf22 were compared with those of other HK. The GenBank accession numbers for the amino acid sequences are: S. coelicolor A3(2) (HisKA-SC, SCO4021), S. avermitilis MA-4680 (HisKA-SA, SAV4197) and S. clavuligerus (Orf22, EU594508). The H-box consensus sequences are a putative phosphorylation site.,$+ \mathrm{N}$ and $\mathrm{G}$ denote the position of the conserved positive amino acid residue. The conserved motifs are enclosed in boxes. 


\section{Analysis of CA Production}

A 2-ml sample was taken from each culture once every 12 hours during the fermentation process. The supernatant was used for assays of CA [19 21]. CA was analyzed by reaction with imidazole [22]. The filtered fermentation supernatant $(90 \mathrm{ml})$ was reacted with $30 \mathrm{ml}$ of $3.0 \mathrm{M}$ imidazole solution $(\mathrm{pH} 6.8)$ at $37^{\circ} \mathrm{C}$ for 40 minutes. The imidazole derivative was diluted 15 -fold with $\mathrm{ddH}_{2} \mathrm{O}$, and its absorbance was measured at $312 \mathrm{~nm}$ [23]. CA yields in S. clavuligerus strains were determined by comparison to a calibration curve generated from a pure standard of CA. CA was also analyzed by high-performance liquid chromatography (HPLC) using a C18 reversed-phase column $(4.6 \times 250 \mathrm{~mm}, 50 \mu \mathrm{m}$ KANTO Reagents, Japan) at $312 \mathrm{~nm}$. The mobile phase consisted of $0.1 \mathrm{M} \mathrm{KH}_{2} \mathrm{PO}_{4}(\mathrm{pH}$ 3.3 adjusted with $\mathrm{H}_{3} \mathrm{PO}_{4}$ ) and $6.0 \% \mathrm{MeOH}$ (flow rate $1.0 \mathrm{ml} / \mathrm{minute}$ ). Thus, the mycelium dry weight of the $S$. clavuligerus/Aorf23 and the wild-type were determined at different incubation times in R2YE media. Mycelium in $50 \mathrm{ml}$ culture was harvested by centrifugation at $6,000 \mathrm{rpm}$ for 17 minutes. The mycelium was washed with $\mathrm{ddH}_{2} \mathrm{O}$ and then dried at $80^{\circ} \mathrm{C}$ to invariable weight.

\section{Results}

\section{Sequence Information of New CA-Related Genes}

It has been previously reported that a series of partial structural genes are involved in the CA biosynthetic pathway [24]. While these genes accounted for many of the expected activities, it was not clear that all of the genetic information needed to form CA was present in this region. In view of the possibility that additional genes might be required for the production of $\mathrm{CA}$, we investigated the DNA sequence of the region beyond this previously reported area. For the investigation of additional genes, a cosmid library was cross-hybridized with a cas2-labeled probe under highly stringent conditions. Colony hybridization, restriction analysis, and Southern hybridization of the probe with the BamHI-digested cosmid DNA of the positive clone led to the identification of clone pCLVA9. Complete sequence analysis of pCLVA9 revealed a 43,889-bp segment that consisted of nine new putative ORFs downstream of the genes for the CA biosynthetic pathway. These analyses are summarized in Fig. 1 and Table 1. These nine new ORFs have been deposited in GenBank under the accession number EU594508.

\section{Sequence Analysis of $\operatorname{orf} 22$ and $\operatorname{orf} 23$}

The $\operatorname{orf} 22$ and $\operatorname{orf} 23$ genes are adjacent to each other on the chromosome, and they are transcribed in the same orientation. The stop and start codons of orf22 and orf23, respectively, overlap by four base pairs (data not shown). The orf 22 coding region is $1,716 \mathrm{bp}$ in length and encodes 571 aa, with a predicted molecular mass of $59.7 \mathrm{kDa}$. The deduced protein sequence of Orf22 shows the typical

B.

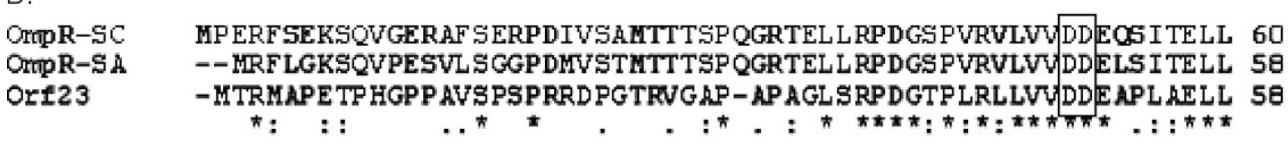

OMPR-SC SMALRYEGUQIRS AGDGHGAVQTARDFRPDAVUIDMML PDWDGL SVLGRL RRDLPDVPVL 120 MTR-SA SMALRYEGUOIRS AGDGQGAVQTAREFRPDAVVL DMML PD MDGL TVLGRL RRELPDVPVL 118 Orf23 SMAL RYEGUEYRSAGD GSTAVRT AREFRPDAVVL DYML PDMDGL TVLARL RRERPEVPVL 110

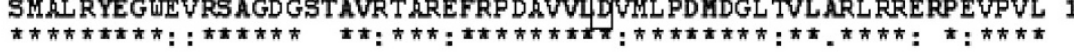

OMPR-SC FLTAKDAVEDRIAGLTAGGDDYVTKPFSLEEVVARLRGLIRRSGAADRRSDSVLVVGDLT 180 OMpR-SA FLTKKDAVEDRIAGLT TGGDDYYTKPFSLEEVVARLRGLIRRSGAADRRSDSMLVVGDLT 179 Orf23 FLTKRDAVEDRIAGLTAGGDDYYKRFSLEEVVVRLRGLLRRSGAVSARPDTVLVVGDLT 170

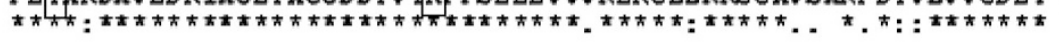

OMPR-SC LDEDSHEVTRSGDGIHL TATEF ELLRFL MRNPRRVLS KAQILDRVUSYDF GGQANVVELY 240 OTtpR-SA LDEDSHEVSRSGENIHL TATEF ELLRFL MRNPRRVLSKAQILD RUUSYD F GGQANVUELY 230 Orf23 LDEESHEVSRGGQA IRL TATEF ELLRCL MRS PRRVLSKAQILDRUWSYDF GGQANVUE IY 230

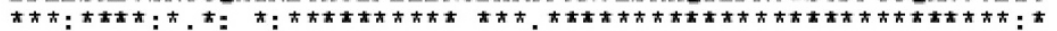

ORTR-SC IS YLRRKIDAGREPMIHTRRGAGYL IKPAVS- 271 OMR-SA ISYLRRKIDAGREPMIHTRRGAGYL IKPAYS- 269 OIf23 ISYLRRKIDAGRAPMIHTRRGAGYLLIPAEGT 270

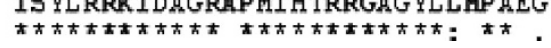

Fig. 2 Amino acid sequence alignment of the TCS-HK (orf22) and TCS-RR (orf23). (Continued)

B. The deduced amino acid sequences of the S. clavuligerus Orf23 were compared with those of other TCS-RR. The GenBank accession numbers for the amino acid sequences are: S. coelicolor A3(2) (OmpR-SC, SCO4020), S. avermitilis MA-4680 (OmpR-SA, SAV4198) and S. clavuligerus (Orf23, EU594508). 
characteristics of prokaryotic HK (Fig. 2A). Orf22 showed $49 \%$ identity with the putative TCS-HK of S. coelicolor A3(2) (HisKA-SC, SCO4021) and 43\% identity with TCSHK of $S$. avermitilis MA-4680 (HisKA-SA, SAV4197). In addition, DNA sequencing showed that the orf 23 coding region is $813 \mathrm{bp}$ in length and encodes $270 \mathrm{aa}$, with a predicted molecular mass of $29.5 \mathrm{kDa}$, and that it belongs to the RR of the OmpR family [3]. The predicted amino acid sequence of $S$. clavuligerus Orf23 was compared with those of other bacteria. The deduced amino acid sequence showed $73 \%$ identity with the putative TCS-RR of $S$. avermitilis MA-4680 (OmpR-SA, SAV4198) and 71\% identity with the putative TCS-RR of S. coelicolor A3(2) (OmpR-SC, SCO4020) (Fig. 2B).

\section{Disruption of the orf 23 and Analysis of CA Production}

The pHN2 containing the orf23-disrupted cassette was transformed into $S$. clavuligerus using a protoplast transformation method. After plasmid curing, four putative mutants (S. clavuligerus/Aorf23) that were apramycinsensitive and neomycin-resistant were selected. Insertional inactivation of orf 23 via double-crossover was verified by Southern blot hybridization using the DNA fragment from the downstream region of the orf22 as the probe (Fig. 3). As shown in Fig. 3B, a $4.67 \mathrm{~kb}$ hybridizing fragment was found in the wild-type, while a $2.68 \mathrm{~kb}$ hybridizing fragment was found in the double-crossover mutant, thus indicating the insertion of the neo gene.

The effect of $\operatorname{orf} 23$ on CA production was determined by using the wild-type, S. clavuligerus/Aorf 23 and $S$.
clavuligerus/Dorf23/pICA1 as batch cultures in CA production media. After 48 hours incubation, samples were withdrawn every 12 hours in order to assess the yield of secondary metabolites for HPLC analysis (data not shown). CA production increased initially from 48 hours becomes more and more pronounced as time period increases, ultimately reached to the highest CA production at 60 hours. On reaching to the highest saturated level, it declines continually showing a slight decrease of CA production at 72 hours (Fig. 4A). Also, CA production of the disrupted mutants was remarkably lower rather than that of the wildtype, and the complemented mutants showed restoration of CA production nearly $80 \%$ (Fig. 4A).

\section{Enhancement of CA Production Using Overexpression of $\operatorname{orf} 22$ and $\operatorname{orf} 23$}

We constructed recombinants, pICA1 (orf22/orf23) in pIBR25 containing a strong ermE $^{*}$ promoter. The growth curve was plotted by variation of mycelium dry weight (g/liter) versus time period (hour) (Fig. 4B). Growth rates of different overexpressed samples repetitively showed higher as compared with that of wild-type, and the biomass of both wild-type and orf23-disrupted mutant was also observed, found to be nearly same with no morphological change. Thus, as shown in Fig. 4A, the co-overexpression of both orf 22 and $\operatorname{orf} 23$ encoding a TCS HK/sensor kinase and RR, respectively, enhanced 1.49 -fold overproduction of CA at 60 hours compared with that of $S$. clavuligerus/pIBR25. These results suggest that the overexpression of orf22/orf 23 enhances CA production.
A.

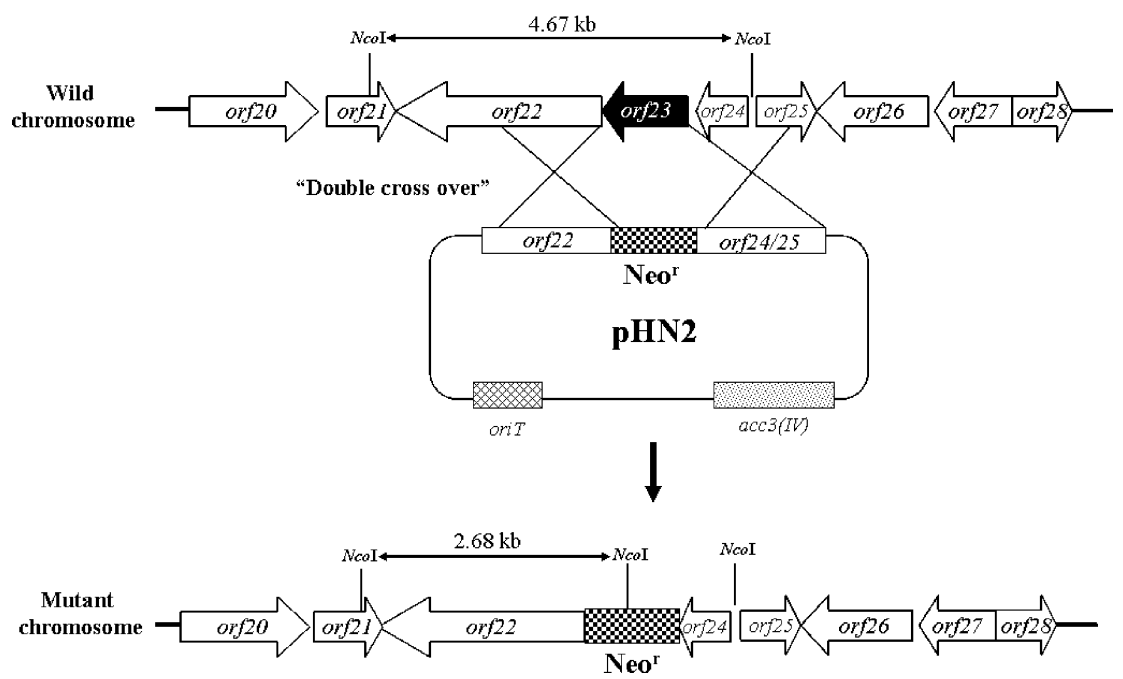

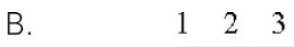

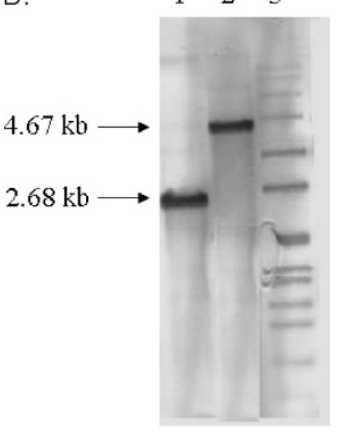

Fig. 3 Generation of orf23-deleted mutants (S. clavuligerus/Aorf23) via double-crossover integration of $\mathrm{pHN2}$ (A) and Southern analysis of genomic DNA from wild-type and orf23-deleted mutants after Ncol digestion (B). 


\section{Discussion}

Some regulatory genes have been previously characterized for their regulatory roles in antibiotic biosynthesis in $S$. clavuligerus. The ccaR controls the production of both cephamycin C and CA [7], and the claR only encodes a LysR-type regulatory protein controlling only CA production [8, 9]. In addition, several TCS containing AfsQ1/Q2 [25], CutR/S [26], PhoP/R [27], EcrA1/A2 [28] and RapA1/A2 [29] have been previously characterized for their regulatory roles in antibiotic biosynthesis in various Streptomyces species. So far, no TCS-related genes involved in the regulation of CA biosynthesis have been reported from $S$. clavuligerus.

Analysis of the deduced protein products of the Orf 23 demonstrated that it possesses the conserved $N$-terminal motifs that are characteristic of other RR. Like other RR, the site of phosphorylation in an aspartate residue is located at the $C$-terminus. The pocket containing this Asp is referred to as the active site since it is generally accepted that the phosphoryl group is transferred from the sensor kinase to the RR [30]. It comprises a constellation of five residues that are strongly conserved across the RR family, shown in Fig. 2B [31]. The deduced protein product of Orf22 exhibits similarity to members of the EnvZ family of HK, including the H-box (His-283 being the putative phosphorylation site) and the $\mathrm{N}$ and $\mathrm{G}$ motifs [32, 33] (Fig. 2A).

Genes involved in the biosynthesis of CA have been located in the $S$. clavuligerus gene cluster, but still some steps in the proposed biosynthetic pathway have no corresponding genes in this cluster. In this study, the DNA sequence of the $S$. clavuligerus CA gene cluster was extended, and also mutants from flanking regions were prepared and analyzed to assess their involvement in CA biosynthesis. Although growth and morphological analyses showed no difference between the knockout mutant and wild-type, a visible decrease of CA production was

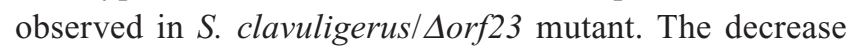
can be restored by introducing pICA1. In addition we have reported for the first time that $\mathrm{CA}$ is regulated by TCSHK/RR (Orf22/23) in the CA biosynthetic pathway. Disruption, complementation and overexpression of the $\operatorname{orf} 23$ demonstrated that it might function as a positive regulator in the biosynthesis of CA. The effects of various environmental factors on the TCS-mutant $(S$.

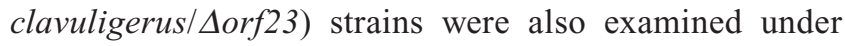
various growth conditions. Characterization of the insertion mutants revealed no phenotypic differences from the wild-type. Response regulators involved in antibiotic
A.

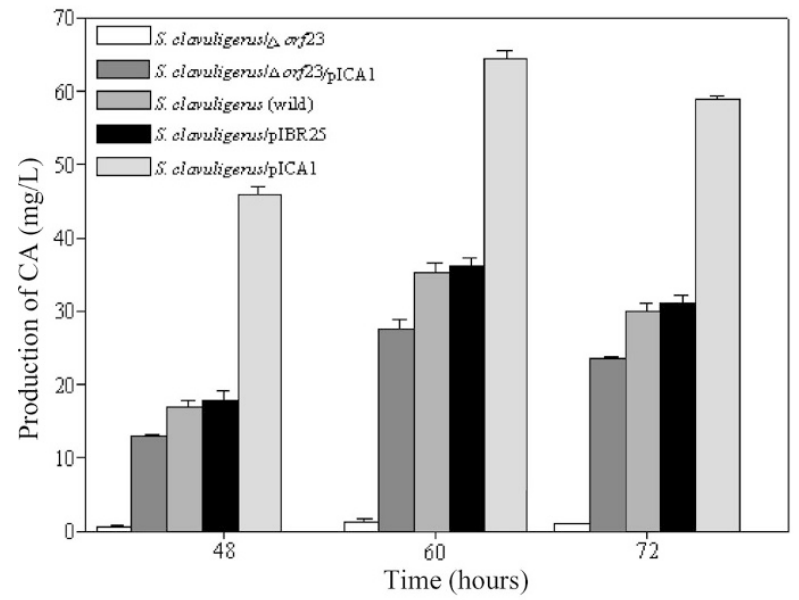

B.

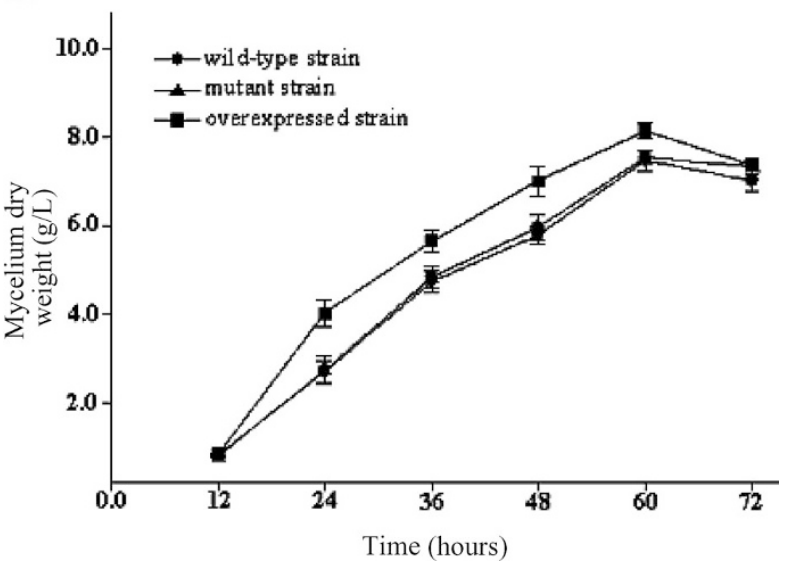

Fig. 4 Comparison of CA products (A) and dry weights (B) from wild-type and mutants.

Measurement of CA products at different growth stages (exponential phase, 48 hours; stationary phase, 60 hours; phase of

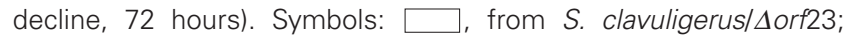
$\square$, from $S$. clavuligerus/Dorf23/pICA1; $\square$, from $S$. clavuligerus; $\square$, from S. clavuligerus/plBR25; and $\square$, from $S$. clavuligerus/pICA1 harboring the orf22 and orf23.

biosynthesis are often located within their biosynthetic gene clusters; the discovery of $\operatorname{orf} 22$ and $\operatorname{orf} 23$ downstream of the genes for CA biosynthesis is consistent with this pattern, although it is still unknown how the TCS activates the CA biosynthetic genes.

In this study, we manipulated $\operatorname{orf} 22$ and $\operatorname{orf} 23$ through overexpression in $S$. clavuligerus. Transformants carrying multiple copies of orf22/orf 23 showed enhanced production of CA during fermentation. Gene manipulation with orf $22 /$ orf 23 played important part in this study because the effects of $\operatorname{orf} 22 / \operatorname{orf} 23$ on CA production have not been investigated previously yet. We suggests that the 
overexpressed genes (orf22/orf23) may be promoted by strong promoter $\mathrm{ermE}^{*}$ which activate the cascade CA production. The stimulatory effect on $\mathrm{CA}$ production may be suggested some activation of other genes by overexpression of $\operatorname{orf22/orf23.~Similar~type~of~results~also~}$ found in the claR overexpression in S. clavuligerus [8]. Growth rate also may be another factor to increase CA production because the overexpressed samples showed little higher growth rate as compared with that of wild-type (Fig. $4 b)$. These results indicated that increased levels of orf22/orf 23 can contribute to enhanced CA biosynthesis.

The production of antibiotics in Streptomyces species generally depends on the growth phase and involves the expression of physically clustered regulatory and biosynthetic genes. The regulatory network is very complicated, thus a thorough analysis of the regulatory network structure is essential for a complete understanding of the gene regulatory pattern and morphology, including physiological development, in Streptomyces and other complex microorganisms. Ultimately, it will provide new strategies for manipulating secondary metabolism and for increasing the production of valuable biologically active natural products.

Acknowledgments The work was supported by a medium-term strategic technology development grant from the Republic of Korea.

\section{References}

1. Ogura M, Tanaka T. Recent progress in Bacillus subtilis two-component regulation. Fron Biosci 7: d1815-d1824 (2002)

2. Bijlsma JJ, Groisman EA. Making informed decisions: regulatory interactions between two-component systems. Trends Microbiol 11: 359-366 (2003)

3. Hutchings MI, Hoskisson PA, Chandra G, Buttner MJ. Sensing and responding to diverse extracellular signals? Analysis of the sensor kinases and response regulators of Streptomyces coelicolor A3 (2). Microbiology 150: 2795-2806 (2004)

4. Fabret C, Feher VA, Hoch JA. Two-component signal transduction in Bacillus subtilis: how one organism sees its world. J Bacteriol 181: 1975-1983 (1999)

5. Mendes MV, Tunca S, Anton N, Recio E, Sola-Landa A, Aparicio JF, Martın JF. The two-component phoR-phoP system of Streptomyces natalensis: Inactivation or deletion of phoP reduces the negative phosphate regulation of pimaricin biosynthesis. Metab Eng 9: 217-227 (2007)

6. Liras P, Rodriguez-Garcia A. Clavulanic acid, a betalactamase inhibitor: biosynthesis and molecular genetics.
Appl Microbiol Biotechnol 54: 467-475 (2000)

7. Perez-Llarena FJ, Liras P, Rodriguez-Garcia A, Martin JF. A regulatory gene $(c c a R)$ required for cephamycin and clavulanic acid production in Streptomyces clavuligerus: amplification results in overproduction of both $\beta$-lactam compounds. J Bacteriol 179: 2053-2059 (1997)

8. Perez-Redondo R, Rodríguez-García A, Martín JF, Liras P. Deletion of the $p y c$ gene blocks clavulanic acid biosynthesis except in glycerol-containing medium: Evidence for two different genes in formation of the C3 unit. J Bacteriol 181: 6922-6928 (1999)

9. Hung TV, Malla S, Park BC, Kiou K, Lee HC, Sohng JK. Enhancement of clavulanic acid by replicative and integrative expression of ccaR and cas 2 in Streptomyces clavuligerus NRRL3585. J Microbiol Biotechnol 17: 1538-1545 (2007)

10. Song JY, Kim ES, Kim DW, Jensen SE, Lee KJ. Functional effects of increased copy number of the gene encoding proclavaminate amidino hydrolase on clavulanic acid production in Streptomyces clavuligerus ATCC 27064. J Microbiol Biotechnol 18: 417-426 (2008)

11. Kieser T, Bibb MJ, Buttner MJ, Chater KF, Hopwood DA. Practical Streptomyces Genetics. John Innes Foundation 2nd: (2000)

12. Sthapit B, Oh TJ, Rajan L, Liou KK, Lee HC, Kim CG, Sohng SK. Neocarzinostatin naphthoate synthase: an unique iterative type I PKS from neocarzinostatin producer Streptomyces carzinostaticus. FEBS Lett 566: 201-206 (2004)

13. Sambrook J, Russell DW. Molecular Cloning: A Laboratory Manual. Cold Spring Harbor Laboratory Press, Cold Spring Harbor, NY (2001)

14. Ishikawa J, Hotta K. Frame plot: A new implementation of the frame analysis for predicting protein coding regions in bacterial DNA with high $\mathrm{G}+\mathrm{C}$ content. FEMS Microbiol Lett 174: 251-253 (1999)

15. Altschul SF, Gish W, Miller W, Myers EW, Lipman DJ. Basic local alignment search tool. J Mol Biol 215: 403-441 (1990)

16. Thompson JD, Higgins DG, Gibson TJ. Clustal W: Improving the sensitivity of progressive multiple sequence alignment through sequence weighting, position-specific gap penalties and weight matrix choice. Nucleic Acids Res 22: 4673-4680 (1994)

17. Garcia-Dominguez M, Martin JF, Mahro B, Demain AL, Liras P. Efficient plasmid transformation of the $\beta$-lactam producer Streptomyces clavuligerus. Appl Environ Microbiol 53: 1376-1381 (1987)

18. Madduri K, Hutchinson CR. Functional characterization and transcriptional analysis of the $d n r R l$ locus, which controls daunorubicin biosynthesis in Streptomyces peucetius. J Bacteriol 177: 1208-1215 (1995)

19. Aoki H, Sakai H, Kohsaka M, Konomi T, Hosoda J. Nocardicin A, a new monocyclic $\beta$-lactam antibiotic. I. Discovery, isolation and characterization. J Antibiot 29: 
492-500 (1976)

20. Pruess DL, Kellett M. Ro-22-5417, a new clavam antibiotic from Streptomyces clavuligerus. I. Discovery and biological activity. J Antibiot 36: 208-212 (1983)

21. Romero J, Liras P, Martin JF. Dissociation of cephamycin and clavulanic acid biosynthesis in Streptomyces clavuligerus. Appl Microbiol Biotechnol 20: 318-325 (1984)

22. Bird AE, Bellis JM, Gasson BC. Spectrophotometric assay of clavulanic acid by reaction with imidazole. Analyst 107: 1241-1245 (1982)

23. Li R, Townsend CA. Rational strain improvement for enhanced clavulanic acid production by genetic engineering of the glycolytic pathway in Streptomyces clavuligerus. Metab Eng 8: 240-252 (2006)

24. Jensen SE, Paradkar AS, Mosher RH, Anders C, Beatty PH, Brumlik MJ, Griffin A, Barton B. Five additional genes are involved in clavulanic acid biosynthesis in Streptomyces clavuligerus. Antimicrob Agents Chemother 48: 192-202 (2004)

25. Ishizuka H, Horinouchi S, Kieser HM, Hopwood DA, Beppu T. A putative 2-component regulatory system involved in secondary metabolism in Streptomyces spp. J Bacteriol 174: 7585-7594 (1992)

26. Chang HM, Chen MY, Shieh YT, Bibb MJ, Chen CW. The cutRS signal transduction system of Streptomyces lividans represses the biosynthesis of the polyketide antibiotic actinorhodin. Mol Microbiol 21: 1075-1085 (1996)

27. Sola-Landa A, Moura RS, Martin JF. The two-component PhoR-PhoP system controls both primary metabolism and secondary metabolite biosynthesis in Streptomyces lividans. Proc Natl Acad Sci USA 100: 6133-6138 (2003)

28. Li YQ, Chen PL, Chen SF, Wu D, Zheng J. A pair of two component regulatory genes ecrA1/A2 in Streptomyces coelicolor. J Zhejiang Univ Sci 5: 173-179 (2004)

29. Lu Y, Wang W, Shu D, Zhang W, Chen L, Qin Z, Yang S, Jiang W. Characterization of a novel two-component regulatory system involved in the regulation of both actinorhodin and a type I polyketide in Streptomyces coelicolor. Appl Microbiol Biotechnol 77: 625-635 (2007)

30. Lewis RJ, Brannigan JA, Muchova K, Barak I, Wilkinson AJ. Phosphorylated aspartate in the structure of a response regulator protein. J Mol Biol 294: 9-15 (1999)

31. Lewis RJ, Muchova K, Brannigan JA, Barak I, Leonard G, Wilkinson AJ. Domain swapping in the sporulation response regulator Spo0A. J Mol Biol 297: 757-770 (2000)

32. Perez-Redondo R, Rodriguez-Garcia A, Martin JF, Liras P. The claR gene of Streptomyces clavuligerus, encoding a LysR-type regulatory protein controlling clavulanic acid biosynthesis, is linked to the clavulanate-9-aldehyde reductase (car) gene. Gene 211: 311-321 (1998)

33. Kim D, Forst S. Genomic analysis of the histidine kinase family in bacteria and archaea. Microbiology 147: 1197-1212 (2001) 